\title{
Electron transport in iodide-capped core@shell PbTe@PbS colloidal nanocrystal solids 루
}

\author{
Cite as: Appl. Phys. Lett. 117, 173101 (2020); doi: 10.1063/5.0025965 \\ Submitted: 19 August 2020 - Accepted: 12 October 2020 . \\ Published Online: 26 October 2020
}
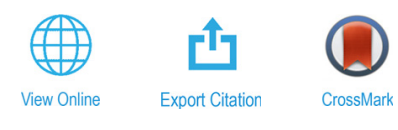

Retno Miranti, (D) Ricky Dwi Septianto,, 2 (D) Maria Ibáñez, ${ }^{3}$ (D) Maksym V. Kovalenko, ${ }^{4,5}$ (D) Nobuhiro Matsushita, Yoshihiro Iwasa, ${ }^{2,6}$ (D) and Satria Zulkarnaen Bisri ${ }^{1,2, a)}$ (D)

\begin{abstract}
AFFILIATIONS
'Department of Materials Science and Engineering, Tokyo Institute of Technology, 2-12-1 Ookayama, Meguro, Tokyo 152-8550, Japan ${ }^{2}$ RIKEN Center for Emergent Matter Science (CEMS), 2-1 Hirosawa, Wako, Saitama 351-0198, Japan

${ }^{3}$ Institute of Science and Technology Austria, Am Campus 1, 3400 Klosterneuburg, Austria

${ }^{4}$ Department of Chemistry and Applied Biosciences, ETH Zurich, Vladimir Prelog Weg 1, Zurich 8093, Switzerland

${ }^{5}$ EMPA-Swiss Federal Laboratories for Materials Science and Technology, Uberlandstrasse 129, Dubendorf 8600, Switzerland

${ }^{6}$ Quantum Phase Electronic Center (QPEC) and Department of Applied Physics, The University of Tokyo, 7-3-1 Hongo, Bunkyo-ku, Tokyo 113-8656, Japan
\end{abstract}

a) Author to whom correspondence should be addressed: satria.bisri@riken.jp

\begin{abstract}
Research in the field of colloidal semiconductor nanocrystals (NCs) has progressed tremendously, mostly because of their exceptional optoelectronic properties. Core@shell NCs, in which one or more inorganic layers overcoat individual NCs, recently received significant attention due to their remarkable optical characteristics. Reduced Auger recombination, suppressed blinking, and enhanced carrier multiplication are among the merits of core@shell NCs. Despite their importance in device development, the influence of the shell and the surface modification of the core@shell NC assemblies on the charge carrier transport remains a pertinent research objective. Type-II PbTe@PbS core@shell NCs, in which exclusive electron transport was demonstrated, still exhibit instability of their electron transport. Here, we demonstrate the enhancement of electron transport and stability in PbTe@PbS core@shell NC assemblies using iodide as a surface passivating ligand. The combination of the PbS shelling and the use of the iodide ligand contributes to the addition of one mobile electron for each core@shell NC. Furthermore, both electron mobility and on/off current modulation ratio values of the core@shell NC field-effect transistor are steady with the usage of iodide. Excellent stability in these exclusively electron-transporting core@shell NCs paves the way for their utilization in electronic devices.
\end{abstract}

Published under license by AIP Publishing. https://doi.org/10.1063/5.0025965

In recent years, colloidal semiconductor nanocrystals (NCs) with core@shell structures attract much greater attention for developing solution-processable optoelectronic and energy harvesting devices. ${ }^{1,2}$ Overcoating individual NCs with different inorganic shells or modifying several atomic layers of the outer surface of the NCs (e.g., via the cation exchange process) has been found as a method to enhance many of the beneficial quantum-confinement related properties. For example, some of the core@shell NC structures that form the so-called type-I core@shell are known to have significantly suppressed nonradiative Auger recombination. ${ }^{3}$ Having the conduction and valence bands of the shell wrap those of the core, they can possess much-enhanced luminescence efficiency, which is beneficial for many applications such as displays and lightings. ${ }^{4-6}$ In addition to demonstrating lasing actions, ${ }^{7,8}$ some of them also demonstrated suppressed photoluminescence blinking/intermittency, which is vital for their prospective use as biolabels/fluorophores and reliable single-photon emitters in quantum computing and quantum communications applications..$^{9-12}$ The reduced density of surface traps due to shelling has been a commonplace strategy to boost the photovoltaic characteristics of quantumconfined absorber materials. ${ }^{13-15}$ Furthermore, the staggered band structure formed in the type II core@shell resulted in the spatial separation of the hole and electron in a different region, enabling the fast transfer of an electron from NCs to the surrounding system beneficial for sensitizer and bioimaging applications. ${ }^{16,17}$ The merits of core@shell structures have mostly been demonstrated from their optical and photonics properties' viewpoints. However, the influence of the shells and their surface on charge carrier transport is comparatively less represented in the literature. This lack of knowledge hampers the realization 
of improved photovoltaic, photodetector, and direct electroluminescence devices, in which core@shell NCs also act as charge transporting mediums.

Recently, we demonstrated the robust control of charge carrier transport in the assemblies of PbTe@PbS core@shell NCs. ${ }^{18}$ Both $\mathrm{PbTe} \mathrm{NCs}$ and $\mathrm{PbS}$ NCs are known to support ambipolar charge carrier transport, that is, where both holes and electrons can be transported from one NC to another. ${ }^{19-21}$ Shelling a PbTe NC core with $\mathrm{PbS}$ made them exclusively electron-transporting. The energy level offset in a so-called quasi-type-II NC made the hole strongly localized within the NC. At the same time, the shell acted as an electron dopant, thus enhancing its electronic conductivity. It was among the first demonstrations of charge carrier transport in core@shell NC assemblies that utilized conventional organic ligands. Previously, electron transport was only demonstrated in the CdSe@CdS core@shell NC assembly that utilized a molecular metal-chalcogenide-complex (MCC) as the capping ligand, without complete understanding regarding the fate of the hole transport in the system. ${ }^{22,23}$ It was only expected that the specific reported configuration might also behave as type-II NCs although many other reports on their optical characteristics mostly exhibit type-I NCs. ${ }^{10,24}$ The demonstration of electron transport with complete suppression of hole transport in the narrow bandgap PbTe@PbS core@shell NC paves ways for its utilization in energy harvesting devices where electron transport is vital, such as thermoelectric and photovoltaics. ${ }^{25,26}$ Furthermore, a quasi-type-II NC was expected to show the enhanced carrier multiplication $(\mathrm{CM})$ process beneficial for photovoltaics. ${ }^{27}$

Although core@shell NCs are expected to be more stable toward the environment than their core NC, the surface of the PbTe@PbS core@shell NC is still sensitive. It affects the electron transport in the assemblies. Both PbTe NCs and PbS NCs are sensitive to oxidation and physisorption of diverse atoms and molecules that hamper the electron transport due to the creation of mid-gap electron trap states. ${ }^{28}$ The surface state of PbTe@PbS NCs can impart similar effects. Therefore, it is vital to treat the surface of the core@shell NCs with appropriate passivating capping molecules to alleviate this problem and enhance their electron transport stability.

Here, we demonstrate the enhancement of electron transport in the assemblies of core@shell PbTe@PbS colloidal NCs and their stability by utilizing iodide as the surface passivating ligand. We measure the electron transport of the core@shell NC assemblies by utilizing them as field-effect transistor (FET) channels. Furthermore, the timedependent electron transport stability of the devices is observed. We compared two different groups of FETs that utilized PbTe@PbS core@shell NC assemblies as their active channel. The first set of devices uses iodide as the capping ligand of the nanocrystals, and the second uses the 1,2-ethanedithiol (EDT) ligand [Fig. 1(a)]. Both ligands have been widely used to modify the surface of colloidal NCs, chiefly in core-only Pb-chalcogenide NCs. ${ }^{18,29-32}$ The effective use of the EDT ligand in PbTe@PbS core@shell nanocrystal assemblies to provide conducting transistor channels has been recently demonstrated. ${ }^{18}$ Nevertheless, the stability of the electron transport of the transistor remains poor. The use of EDT as a ligand was known to be ineffective for preventing electron transport degradation upon prolonged exposure of the NC assemblies to air or moisture. ${ }^{28,33}$ In core-only $\mathrm{Pb}$-chalcogenide NCs with the EDT ligand, the n-type or ambipolar transport characteristics would transform to p-type transport due to

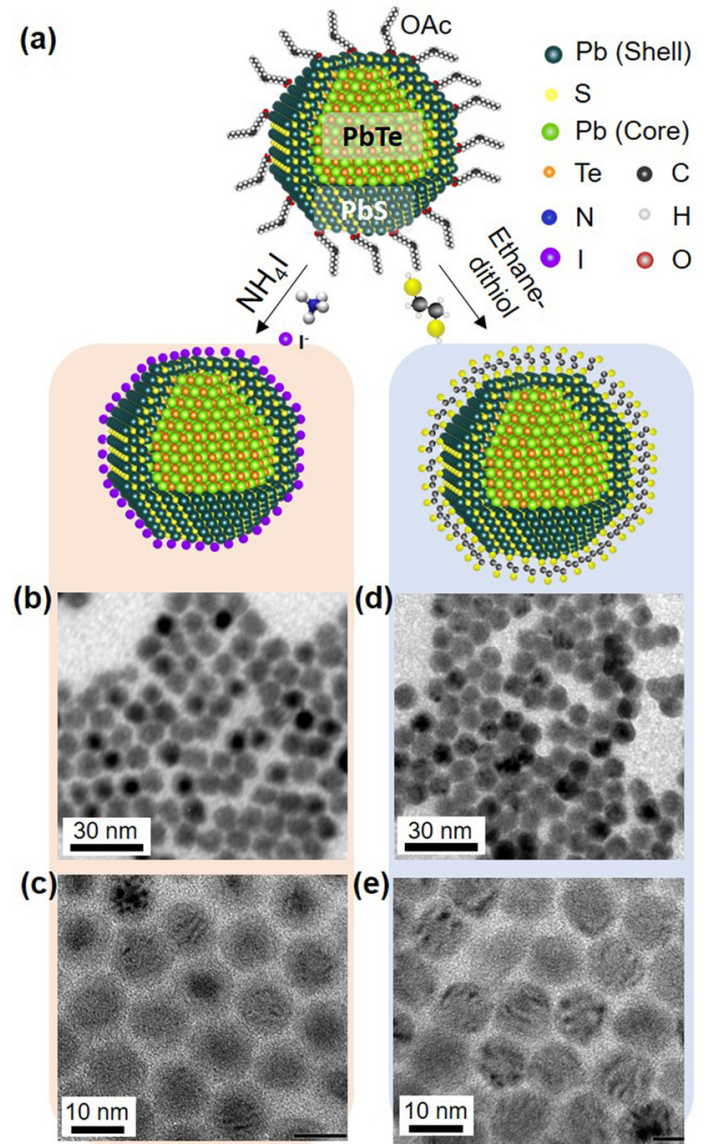

FIG. 1. (a) Schematic of oleic-acid-capped core@shell PbTe@PbS NCs treated by either 1,2-ethanedithiol (EDT) or ammonium iodide $\left(\mathrm{NH}_{4} \mathrm{l}\right)$ to produce either EDTcapped NC or iodide-capped NC. (b) The transmission electron micrograph (TEM) of the iodide-capped PbTe@PbS core@shell NC assembly and (c) their corresponding high-resolution transmission electron micrograph (HRTEM) image. (d) The TEM and (e) the HRTEM images of the EDT-capped PbTe@PbS core@shell NC assembly.

this instability. On the other hand, iodide capping provides higher stability in the conventional core-only NC devices. ${ }^{34,35}$ The comparison of these two groups of PbTe@PbS core@shell nanocrystal FETs enables evaluating the influence of the iodide use on the charge carrier transport in these core@shell NCs.

Core@shell PbTe@PbS NCs with an overall diameter of $11 \mathrm{~nm}$, which consist of $7 \mathrm{~nm}$ PbTe core, were used in this study. They were synthesized by following a method, which was described in our previous report. ${ }^{36}$ The sulfur precursor was added, at room temperature, into the crude solution containing synthesized $\mathrm{PbTe}$ nanocrystals and unreacted $\mathrm{Pb}$ oleate. The solution was heated $\left(80^{\circ} \mathrm{C}\right.$ at $\left.1.5^{\circ} \mathrm{C} / \mathrm{min}\right)$ to form a crystalline PbS shell. The synthesized core@shell PbTe@PbS NCs with native oleic acid ligands were washed following established methods to remove solvent and unreacted reagent, which include centrifugation and redispersion..$^{30,37}$

To form the thin-film assemblies of NCs, we deposit them using a layer-by-layer spin coating process. ${ }^{18,28-30}$ A dispersion of $\mathrm{PbTe} @ \mathrm{PbS} \mathrm{NCs}$ in chloroform $\left(5 \mathrm{mg} \mathrm{m}^{-1}\right)$ was spin-coated on the 
substrate at $4000 \mathrm{rpm}$ for each layer. For the FET structure, we used $230 \mathrm{~nm} \mathrm{SiO} / 2$ Si substrates with pre-patterned interdigitated $\mathrm{Au}$ electrodes. The capacitance of the oxide layer is $15 \mathrm{nF} \mathrm{cm}^{-2}$. The channel length and width of the devices were $10 \mu \mathrm{m}$ and $10 \mathrm{~mm}$, respectively. The FET channels consist of five layers of the spin-coated monolayers. The ligand exchange on the PbTe@PbS core@shell NCs to replace the native insulating oleic acid ligand was performed in the solid-state for each spin-coated layer. To perform solid-state ligand exchange with iodide salts, we prepared a solution of $10 \mathrm{mM}$ ammonium iodide $\left(\mathrm{NH}_{4} \mathrm{I}\right)$ in methanol. We utilized $\mathrm{NH}_{4} \mathrm{I}$ rather than the other iodide salts since the amine counterion was essential in this process to assist a complete stripping of the oleic acid ligand. ${ }^{38}$ Methanol is also known as an effective solvent favoring the desorption of oleic acid from the NC surface. ${ }^{20}$ On the other hand, to fabricate the reference samples using the EDT ligand, we dissolved EDT in acetonitrile $(1 \% \mathrm{v} / \mathrm{v}){ }^{29}$

Figures 1(b) and 1(c) show the transmission electron microscopy (TEM) image of the core@shell NCs after the solid-state ligand exchange with iodide. The average distance between the neighboring NCs reduced to $0.6 \mathrm{~nm}$, from initially $2 \mathrm{~nm}$. The distance-reduced NC assembly still forms a hexagonal-like arrangement. It contrasts the observation previously reported for the usage of the iodide ligand for core-only $\mathrm{PbS} \mathrm{NCs}$. In the PbX core-only NC assembly, squarepacked lattices were formed by ligand exchange with iodide. ${ }^{39,40}$ It is due to the faster exchange of the oleic acid ligand on their $\{100\}$ facets than the other $\{110\}$ and $\{111\}$ facets. The inability of the core@shell $\mathrm{PbTe} @ \mathrm{PbS}$ NCs to form a square lattice after iodide ligand exchange suggests that the crystalline shell of the NCs does not conserve the perfect facet of the core NC. The relatively long remaining distance between the NCs suggests that the iodide only acts as the capping layer of the core@shell NCs. A similar occurrence was also found in the core@shell assembly in which EDT was used. The distance between neighboring NCs reduced to $0.49 \mathrm{~nm}$ but without forming a square lattice assembly [Figs. 1(d) and 1(e)]. It happens although the bidentate EDT ligand is still possible to act as a bridge between the NCs. The HRTEM images of the two assemblies show no distinct cubic lattice facets on the NC surface [Figs. 1(c)-1(e)] despite the fact that the moire patterns that indicate the interference of the two highly crystalline structures of the $\mathrm{PbTe}$ and $\mathrm{PbS}$ are observed.

To perform the electrical measurement, we measured the FETs of the NC assemblies [Fig. 2(a)]. All device fabrication and measurement were performed inside an $\mathrm{N}_{2}$-filled glovebox. The transport characteristics of FETs were measured with a voltage scan speed of $20 \mathrm{~V} \mathrm{~s}^{-1}$. Figure 2(b) shows the $I_{D}-V_{D}$ output characteristics of the iodide-capped PbTe@PbS core@shell FET. The device works as n-type FETs. In the $n$-channel operation $\left(V_{D}>0 \mathrm{~V}, V_{G}>0 \mathrm{~V}\right)$, effective electron accumulation was observed (see hysteresis in Fig. S1). On the other hand, in the $p$-channel operation $\left(V_{D}<0 \mathrm{~V}, V_{G}<0 \mathrm{~V}\right)$, there is no indication of hole accumulation since the drain current depletes by the increase in $\left|V_{G}\right|$. The output characteristics exhibit an Ohmic-like electron injection from the $\mathrm{Au}$ electrodes into the $\mathrm{PbTe} @ \mathrm{PbS} \mathrm{NC}$ assemblies, as indicated by the linear $I_{D}$ in the low $V_{D}$ bias regime $\left(V_{D}<10 \mathrm{~V}\right)$ of the $n$-type operation. The demonstration of exclusive electron transport in the $\mathrm{PbTe} @ \mathrm{PbS} \mathrm{NC}$, which is in contrast to its core-only behavior, is in a firm consistency with our previous report. ${ }^{18}$

To investigate the influence of iodide salts on the electronic transport properties of core@shell $\mathrm{PbTe} @ \mathrm{PbS} \mathrm{NC}$ solids, we performed a direct comparison of the FET performance between those with iodide-capping and those with EDT-capping. Two groups of FET devices with similar numbers of the deposited layers were prepared from the same batch of core@shell NCs. Figure 3(a) shows the comparison of the $I_{D^{-}} V_{D S}$ output characteristics of the FET core@shell $\mathrm{PbTe} @ \mathrm{PbS}$ with either EDT-capping or iodide-capping, at $n$-channel operation $\left(V_{D}>0 \mathrm{~V}\right)$ at the highest applied gate voltage in the measurement $\left(V_{G}=80 \mathrm{~V}\right)$. It implies that the use of iodide as a capping ligand for core@shell PbTe@PbS NCs enhances electron transport. The maximum current achieved in the iodide-capped core@shell $\mathrm{PbTe} @ \mathrm{PbS}$ FETs is about five-times to six-times higher than that of EDT-capped.

Figure 3(b) displays the comparison of $I_{D}-V_{G}$ transfer characteristics of the EDT capped and iodide capped core@shell PbTe@PbS NC FETs in the linear operation regime $\left(V_{D}=5 \mathrm{~V}\right)$. Both transfer characteristics show normally on $n$-type transport behavior with no sign of (a)
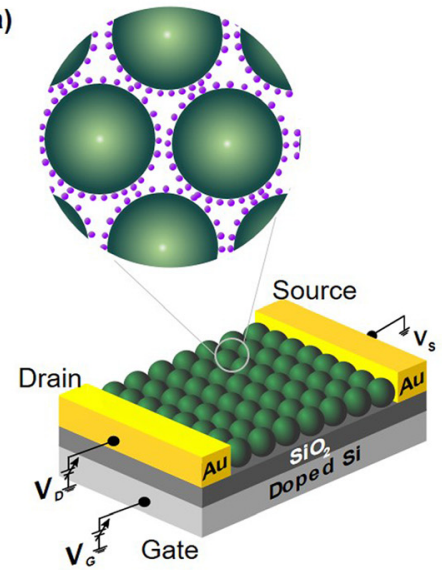

(b) 80

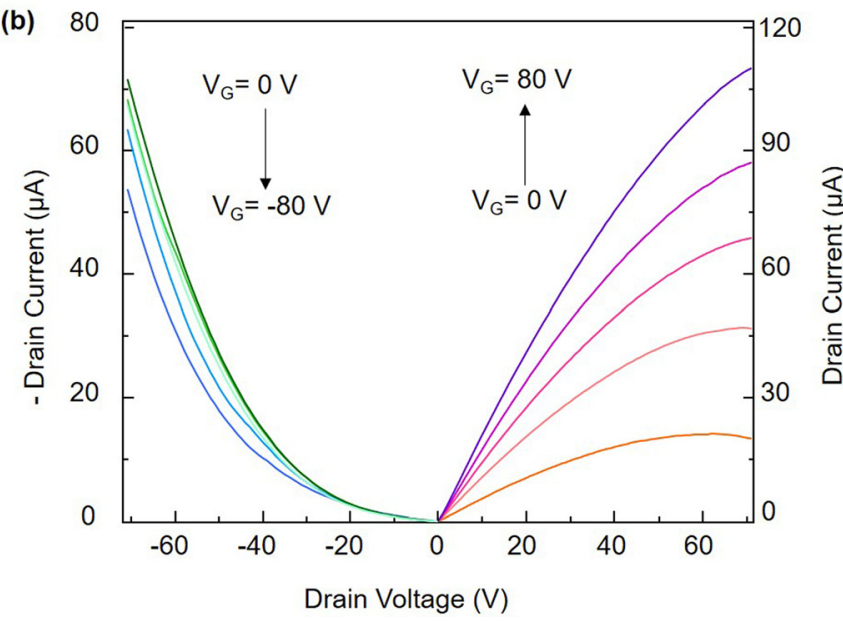

FIG. 2. (a) Schematic of a field-effect transistor (FET) of the iodide-capped core@shell PbTe@PbS NC assembly. (b) The $I_{D}-V_{D}$ output characteristics of the FET in both $p$ channel and $\mathrm{n}$-channel operations. Clear electron accumulations are observed in the n-channel operation. 

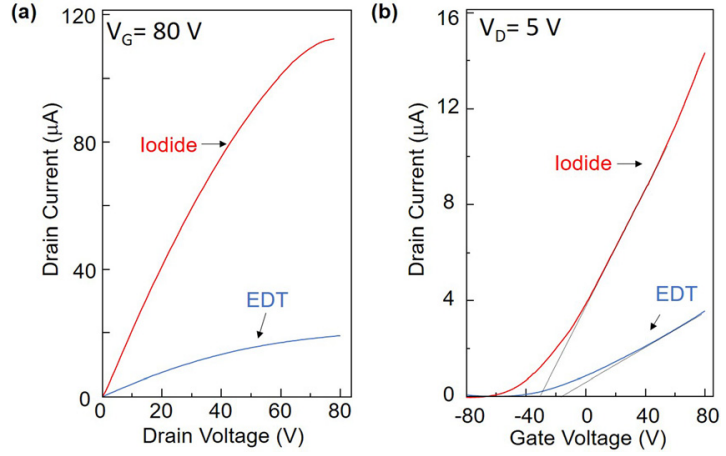

FIG. 3. (a) The comparison of the n-channel $I_{D}-V_{D}$ output characteristics of two different FETs that utilized iodide-capped PbTe@PbS NCs (red-line) and EDT-capped $\mathrm{PbTe} @ \mathrm{PbS}$ NCs (blue-line). The comparison is made at high applied gate voltage $\left(V_{G}=80 \mathrm{~V}\right)$. (b) The comparison of the corresponding n-channel $I_{D}-V_{G}$ transfer characteristics, in the linear regime $\left(V_{D}=5 \mathrm{~V}\right)$. The FET using iodide-capped $\mathrm{PbTe} @ \mathrm{PbS}$ NCs (red-line) shows much higher conductivity and lower electron accumulation threshold voltage $\left(V_{T h}\right)$ than the FET using EDT-capped PbTe@PbS NCs (blue-line).

hole accumulation. The transfer characteristics of the iodide-capped PbTe@PbS FET show much-enhanced electron conductivity with both a steeper curve and a higher maximum electron current than those the EDT-capped device, confirming the observation in the output curve comparison. We extracted electron mobility values of the iodide-capped PbTe@PbS core@shell NC and obtained values up to $2.1 \times 10^{-3} \mathrm{~cm}^{2} \mathrm{~V}^{-1} \mathrm{~s}^{-1}$. This value is much higher than the electron mobility in the EDT-capped PbTe@PbS NC assembly $\left(\mu_{e}=0.6 \times 10^{-3} \mathrm{~cm}^{2} \mathrm{~V}^{-1} \mathrm{~s}^{-1}\right)$.

In the iodide-capped core@shell FET, the gate threshold voltage $\left(V_{T H}\right)$ for electron accumulation was $V_{T H}=-30.2 \mathrm{~V}$, which was deduced from the intercept of the linear extrapolation of the $I_{D^{-}} V_{G}$ transfer curve. On the other hand, the EDT-capped core@shell FET exhibits electron threshold voltage at around $V_{T H}=-16.1 \mathrm{~V}$. This threshold voltage difference is as large as $\left|\Delta V_{T H}\right|=10.3 \mathrm{~V}$ corresponding to $1.32 \times 10^{12}$ electron $/ \mathrm{cm}^{2}$ more in the case of the iodide-capped PbTe@PbS core@shell. The enhancement of electron transport in the iodide-capped PbTe@PbS core@shell is triggered mostly by the passivation of the remaining electron trap states due to the $\mathrm{Pb}$ dangling surface on the $\mathrm{PbS}$ shell. As a consequence, we observed electron-doping-like behavior.

Figure 4(a) shows the comparison of the $I_{D^{-}} V_{G}$ transfer characteristics, which are plotted in the logarithmic scale, of the FETs using the core@shell NC with two different ligands and a FET with EDT-capped PbTe core-only NC (see hysteresis of the transfer characteristics in Fig. S2). The size of the used PbTe NCs is similar to the core diameter in the core@shell NCs. As depicted in this plot, no significant changes were observed in terms of the electron accumulation subthreshold swing value between the FET that used the iodidecapped core@shell NC and the one that used the EDT-capped core@shell NC despite the sharp difference in electron current values. Both devices showed an electron accumulation subthreshold swing value of about $40 \mathrm{~V} /$ decade. It may suggest that the iodide ligand contributes mainly to the passivation of electron deep-trap states instead of the shallower electron trap sites. In addition, the ion polarizability of the ligand molecules (iodide and EDT) is also known to affect the
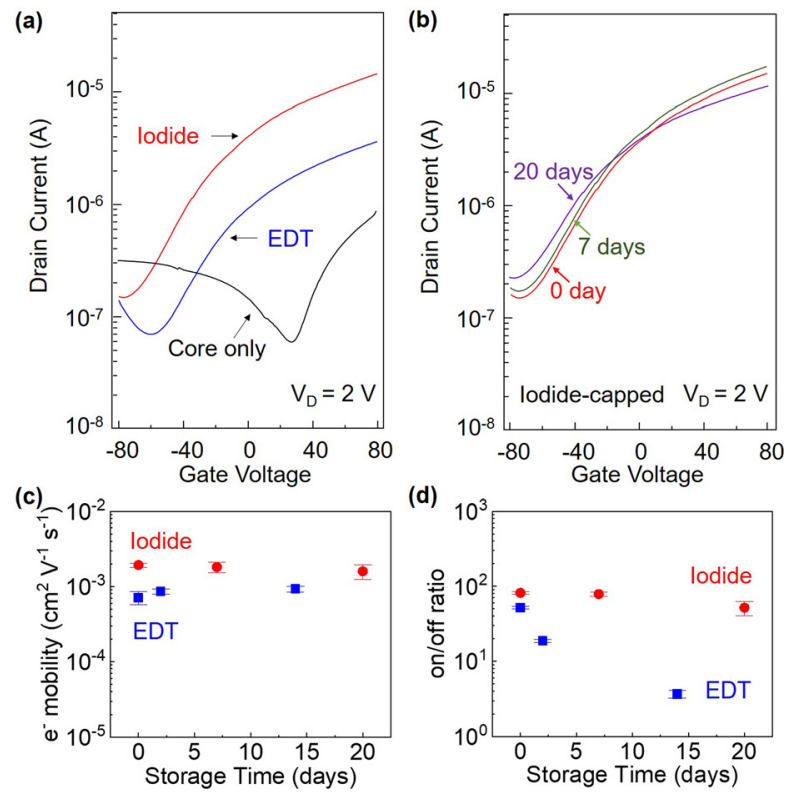

FIG. 4. (a) The logarithmic plot of $n$-channel $I_{D}-V_{G}$ transfer characteristics comparing the FETs using iodide-capped PbTe@PbS NCs (red-line), EDT-capped PbTe@PbS NCs (blue-line), and the EDT-capped PbTe core-only NC assembly (black line) to signify the shift of threshold voltage. (b) The comparison of the $I_{D}-V_{G}$ transfer characteristics of the FETs using iodide-capped PbTe@PbS NCs measured right after device fabrication (red line), after seven days (green line), and after 20 days (purple lines). (c) Time-series plots of the electron mobility and (d) the on/ off current modulation ratio of both the iodide-capped core@shell NC assembly and the EDT-capped core@shell NC assembly suggesting enhanced electron transport stability in the iodide-capped device.

energetic distance between the Fermi level and the conduction band of the NC assemblies. ${ }^{41}$ The minimum change of the subthreshold swing value also reconfirmed that shelling the PbTe NCs with $\mathrm{PbS}$ has significantly modified the electronic energy landscape in which the NCs become a quasi-type II heterojunction system. In comparison to the electronic transport properties of the $\mathrm{PbTe}$ core-only assembly, the combination of $\mathrm{PbS}$ shelling and the usage of iodide capping contributes to the addition of electron density of as much as $1.05 \times 10^{13}$ electron $/ \mathrm{cm}^{2}$. This value was deduced from the difference of electron accumulation threshold voltage of the PbTe core-only FET and the iodide-capped PbTe@PbS core@shell FET, which was about $112.2 \mathrm{~V}$. This value corresponds to about more than one additional mobile electron for each NC.

It should be noted that the large electron subthreshold swing values in both iodide-capped and EDT-capped core@shell FETs are indications of significant electron trapping behavior in the devices, which stemmed from the characteristics of the interface between the $\mathrm{NC}$ assemblies and the oxide gate dielectric. In our previous report, ${ }^{18}$ we demonstrated the use of ionic liquid to form an electric-doublelayer transistor (EDLT) to avoid this interface issue. However, the use of the iodide ligand makes NCs susceptible to get dissolved in the ionic liquid or electrolytes. ${ }^{42}$ It prevents us from measuring the proper EDLT of the iodide-capped PbTe@PbS core@shell NCs. The transistor channel is reproducibly exfoliated and dissolved into the ionic liquid after applying voltages in the measurement. 
To further study the effect of the iodide ion on PbTe@PbS core@shell NCs, we performed the time-series measurement of the FET performance. Figure 4(b) presents the comparison of $I_{D^{-}} V_{G}$ transfer characteristic of an iodide-capped PbTe@PbS NC FET immediately after its preparation and after being stored in an $\mathrm{N}_{2}$ glovebox for seven days and 20 days. The FET exhibits very stable electron transport characteristics, including its hysteresis behavior [Fig. S3(a)]. The only noticeable change is the slight increase in the FET current in the off state, associated with the measurement condition. It should be noted that these NC solids are highly prone to oxidation and sensitive to the physisorption of the even small amount of oxygen and moisture remaining inside the glovebox. Long-term storage of the devices for the measurement typically results in quenching of electron transport, as can also be observed in the EDT-capped NC FETs [Fig. S3(b)].

Figure 4(c) shows the electron mobility trend of iodide-capped and EDT-capped core@shell PbTe@PbS NCs in relation to the storage time. We found that the iodide-capped core@shell NC solids exhibit higher and more stable electron transport characteristics, maintaining the electron mobility value, even after being stored for 7-20 days. The electron mobility of the EDT-capped core@shell NC was also found to be relatively stable. However, the trend of the on/off current modulation ratio of the core@shell PbTe@PbS NC was contrasting between those capped by iodide and those capped by the EDT ligand [Fig. 4(d)]. The on/off modulation ratio in the iodide-capped core@shell device showed stable values, which are entirely different from the values of the EDT-capped core@shell device that is degraded due to the increase in the off-current. It suggests that the usage of the iodide ligand that passivates the remaining $\mathrm{Pb}$ dangling bond prevents the creation of the mid-gap trap states for electrons that usually occur in the EDT-capped NCs, which increases the off-current in the FET operation. These results imply that iodide capping can stabilize the electron transport in the core@shell NC solids in a similar fashion to that in the more conventional core-only Pb-chalcogenide NCs.

In conclusion, we have demonstrated electron transport enhancement and stabilization in the core@shell PbTe@PbS semiconductor $\mathrm{NC}$ assembly by employing the iodide capping ligand. We account for the contribution of the iodide in passivating the remaining $\mathrm{Pb}$ dangling bond on the PbS shell surface, thus enhancing the electron conductivity. Simultaneously, the iodide ligand also acts as a surface passivation agent that prevents both the degradation of the electron transport and the creation of surface-sensitive mid-gap trap states. Therefore, the use of the iodide ligand made the shelf life of the device significantly longer for the electron transport characteristics. The advantage of the exclusive electron transport with stable device performance makes these iodide-capped core@shell PbTe@PbS NCs suitable for practical electronic device applications.

See the supplementary material for information regarding the hysteresis of the plotted transistor characteristics.

This work was partly supported by Grants-in-Aid for Scientific Research by Young Scientist A (KAKENHI Wakate-A) No. JP17H04802, Grants-in-Aid for Scientific Research No. JP19H05602 from the Japan Society for the Promotion of Science, and RIKEN Incentive Research Grant (Shoreikadai) 2016. M.V.K. and M.I. acknowledge financial support from the European Union
(EU) via FP7 ERC Starting Grant 2012 (Project NANOSOLID, GA No. 306733) and ETH Zurich via ETH career seed grant (No. SEED-18 16-2). We acknowledge Mrs. T. Kikitsu and Dr. D. Hashizume (RIKEN-CEMS) for access to the transmission electron microscope facility.

\section{DATA AVAILABILITY}

The data that support the findings of this study are available from the corresponding author upon reasonable request.

\section{REFERENCES}

${ }^{1}$ P. Reiss, M. Protière, and L. Li, Small 5, 154 (2009).

${ }^{2}$ J. M. Pietryga, Y.-S. Park, J. Lim, A. F. Fidler, W. K. Bae, S. Brovelli, and V. I. Klimov, Chem. Rev. 116, 10513 (2016).

${ }^{3}$ J. A. Hollingsworth, Chem. Mater. 25, 1318 (2013).

${ }^{4}$ K.-S. Cho, E. K. Lee, W.-J. Joo, E. Jang, T.-H. Kim, S. J. Lee, S.-J. Kwon, J. Y. Han, B.-K. Kim, B. L. Choi, and J. M. Kim, Nat. Photonics 3, 341 (2009).

${ }^{5}$ E. Jang, S. Jun, H. Jang, J. Lim, B. Kim, and Y. Kim, Adv. Mater. 22, 3076 (2010).

${ }^{6}$ E. Jang, Y. Kim, Y.-H. Won, H. Jang, and S.-M. Choi, ACS Energy Lett. 5, 1316 (2020).

7J. Roh, Y.-S. Park, J. Lim, and V. I. Klimov, Nat. Commun. 11, 271 (2020).

${ }^{8}$ J. Lim, Y. S. Park, and V. I. Klimov, Nat. Mater. 17, 42 (2018).

${ }^{9}$ A. L. Efros and D. J. Nesbitt, Nat. Nanotechnol. 11, 661 (2016).

${ }^{10}$ O. Chen, J. Zhao, V. P. Chauhan, J. Cui, C. Wong, D. K. Harris, H. Wei, H. S. Han, D. Fukumura, R. K. Jain, and M. G. Bawendi, Nat. Mater. 12, 445 (2013).

${ }^{11}$ B. Mahler, P. Spinicelli, S. Buil, X. Quelin, J.-P. Hermier, and B. Dubertret, Nat. Mater. 7, 659 (2008).

${ }^{12}$ A. Nurmikko, Nat. Nanotechnol. 10, 1001 (2015).

${ }^{13}$ D. M. Kroupa, G. F. Pach, M. Vörös, F. Giberti, B. D. Chernomordik, R. W. Crisp, A. J. Nozik, J. C. Johnson, R. Singh, V. I. Klimov, G. Galli, and M. C. Beard, ACS Nano 12, 10084 (2018).

${ }^{14}$ M. J. Speirs, D. M. Balazs, H.-H. Fang, L.-H. Lai, L. Protesescu, M. V. Kovalenko, and M. A. Loi, J. Mater. Chem. A 3, 1450 (2015).

${ }^{15}$ J. E. Murphy, M. C. Beard, A. G. Norman, S. P. Ahrenkiel, J. C. Johnson, P. Yu, O. I. Mićić, R. J. Ellingson, and A. J. Nozik, J. Am. Chem. Soc. 128, 3241 (2006).

${ }^{16}$ Y. Zhang, Y. Li, and X.-P. Yan, Small 5, 185 (2008).

${ }^{17}$ J. Wang, I. Mora-Seró, Z. Pan, K. Zhao, H. Zhang, Y. Feng, G. Yang, X. Zhong, and J. Bisquert, J. Am. Chem. Soc. 135, 15913 (2013).

${ }^{18}$ R. Miranti, D. Shin, R. D. Septianto, M. Ibáñez, M. V. Kovalenko, N. Matsushita, Y. Iwasa, and S. Z. Bisri, ACS Nano 14, 3242 (2020).

${ }^{19}$ S. Z. Bisri, C. Piliego, M. Yarema, W. Heiss, and M. A. Loi, Adv. Mater. 25, 4309 (2013).

${ }^{20}$ L. Liu, S. Z. Bisri, Y. Ishida, T. Aida, and Y. Iwasa, Nanoscale 11, 20467 (2019).

${ }^{21}$ J. J. Urban, D. V. Talapin, E. V. Shevchenko, C. R. Kagan, and C. B. Murray, Nat. Mater. 6, 115 (2007).

${ }^{22}$ J.-S. Lee, M. V. Kovalenko, J. Huang, D. S. Chung, and D. V. Talapin, Nat. Nanotechnol. 6, 348 (2011).

${ }^{23}$ E. Scalise, V. Srivastava, E. Janke, D. Talapin, G. Galli, and S. Wippermann, Nat. Nanotechnol. 13, 841 (2018).

${ }^{24}$ V. Pinchetti, F. Meinardi, A. Camellini, G. Sirigu, S. Christodoulou, W. K. Bae, F. De Donato, L. Manna, M. Zavelani-Rossi, I. Moreels, V. I. Klimov, and S. Brovelli, ACS Nano 10, 6877 (2016).

${ }^{25}$ J. J. Urban, Nat. Nanotechnol. 10, 997 (2015).

${ }^{26}$ M. V. Kovalenko, Nat. Nanotechnol. 10, 994 (2015).

${ }^{27}$ C. M. Cirloganu, L. A. Padilha, Q. Lin, N. S. Makarov, K. A. Velizhanin, H. Luo, I. Robel, J. M. Pietryga, and V. I. Klimov, Nat. Commun. 5, 4148 (2014).

${ }^{28}$ D. M. Balazs, M. I. Nugraha, S. Z. Bisri, M. Sytnyk, W. Heiss, and M. A. Loi, Appl. Phys. Lett. 104, 112104 (2014).

${ }^{29}$ R. D. Septianto, L. Liu, F. Iskandar, N. Matsushita, Y. Iwasa, and S. Z. Bisri, NPG Asia Mater. 12, 33 (2020).

${ }^{30}$ L. Liu, S. Z. Bisri, Y. Ishida, D. Hashizume, T. Aida, and Y. Iwasa, ACS Appl. Nano Mater. 1, 5217 (2018). 
${ }^{31}$ M. I. Nugraha, H. Matsui, S. Watanabe, T. Kubo, R. Häusermann, S. Z. Bisri, M. Sytnyk, W. Heiss, M. A. Loi, and J. Takeya, Adv. Electron. Mater. 3 1600360 (2017).

${ }^{32}$ A. G. Shulga, L. Piveteau, S. Z. Bisri, M. V. Kovalenko, and M. A. Loi, Adv. Electron. Mater. 2, 1500467 (2016).

${ }^{33}$ M. H. Zarghami, Y. Liu, M. Gibbs, E. Gebremichael, C. Webster, and M. Law, ACS Nano 4, 2475 (2010).

${ }^{34}$ D. Zhitomirsky, M. Furukawa, J. Tang, P. Stadler, S. Hoogland, O. Voznyy, H. Liu, and E. H. Sargent, Adv. Mater. 24, 6181 (2012).

${ }^{35}$ Z. Ning, O. Voznyy, J. Pan, S. Hoogland, V. Adinolfi, J. Xu, M. Li, A. R. Kirmani, J.-P. Sun, J. Minor, K. W. Kemp, H. Dong, L. Rollny, A. Labelle, G. Carey, B. Sutherland, I. Hill, A. Amassian, H. Liu, J. Tang, O. M. Bakr, and E. H. Sargent, Nat. Mater. 13, 822 (2014).

${ }^{36}$ M. Ibáñez, R. Zamani, S. Gorsse, J. Fan, S. Ortega, D. Cadavid, J. R. Morante, J. Arbiol, and A. Cabot, ACS Nano 7, 2573 (2013).
${ }^{37}$ C. Piliego, L. Protesescu, S. Z. Bisri, M. V. Kovalenko, and M. A. Loi, Energy Environ. Sci. 6, 3054 (2013).

${ }^{38}$ W. Walravens, J. De Roo, E. Drijvers, S. ten Brinck, E. Solano, J. Dendooven, C. Detavernier, I. Infante, and Z. Hens, ACS Nano 10, 6861 (2016).

${ }^{39}$ D. M. Balazs, B. M. Matysiak, J. Momand, A. G. Shulga, M. Ibáñez, M. V. Kovalenko, B. J. Kooi, and M. A. Loi, Adv. Mater. 30, 1802265 (2018).

${ }^{40}$ D. M. Balazs, D. N. Dirin, H.-H. Fang, L. Protesescu, G. H. ten Brink, B. J. Kooi, M. V. Kovalenko, and M. A. Loi, ACS Nano 9, 11951 (2015).

${ }^{41}$ P. R. Brown, D. Kim, R. R. Lunt, N. Zhao, M. G. Bawendi, J. C. Grossman, and V. Bulović, ACS Nano 8, 5863 (2014).

${ }^{42}$ H. Zhang, K. Dasbiswas, N. B. Ludwig, G. Han, B. Lee, S. Vaikuntanathan, and D. V. Talapin, Nature 542, 328 (2017). 